\title{
Electronic Health Record for Temporomandibular Joint Disorders - Support in Therapeutic Process
}

\author{
R. Hippmann ${ }^{1,2}$, M. Nagy ${ }^{2,3}$, T. Dostálová, ${ }^{1,2}$, J. Zvárová, ${ }^{2,3}$ M. Seydlová, ${ }^{1,2}$ a E. Feltlová \\ 'Department of Paediatric Stomatology, Second Faculty of Medicine of Charles University, Prague, Czech Republic \\ ${ }^{2}$ Centre of Biomedical Informatics Prague, Czech Republic \\ ${ }^{3}$ Institute of Computer Science, Academy of Sciences of the Czech Republic, Prague, Czech Republic, Mathematical Institute AS CR, \\ Prague, Czech Republic
}

Supervisor: Prof. MUDr. Tatjana Dostálová, DrSc., MBA

\section{Summary}

Our experimental work is engaged in creating a new type of Electronic Health Record (EHR) in the field of dentistry, especially for temporomandibular joint (TMJ) disorders (TMD) and the main goal is creation of the comprehensive system, which would be a good basis for the decision support in the TMD therapy.

Our EHR system is based on the Dental knowledge base, which is constructed in the application MUDR (Multimedia Distributed EHR) and then in the MUDRLite application. The MUDR Lite architecture is built on two tiers (relational database (e.g. MS SQL server) and User Interface layer (MUDRLite UI), which is described by an XML configuration file). These applications are creating the real graphical user application DentCross, which is connected with the ASR (Automatic speech recognition) system and TTS (Text-to-speech) module. Special TMD EHR is a part of the Dentcross and is created in the same applications. This part uses the RCD/TMD classification (Research diagnostic criteria for TMD).

We present the Integrated Dental-TMJ EHR system, which allows recording patient's complex medical data in the field of dentistry. It affects the whole orthognathic system with its all components (especially TMD problematic) in the graphical form. The recording and data manipulation are enhanced with the ASR system and TTS module. This application has been constructed with an aim on ease of control and capability of covering medical information and should facilitate decision process in the therapy of TMD.

The integrated Dental - TMJ EHR system represents whole patient's dental information, which is essential for the treatment plan creation. Connection with the ASR system and TTS module simplifies collecting of this information. Information is also well-arranged in the graphical form. Next step is continuation of clinical testing of the whole system and improvement of the ASR system and TTS module. The disadvantage of this system could be necessity of experience in operating with this system and ASR system functionality in the noisy background.

Keywords: electronic health record, automatic speech recognition, dental cross, temporomandibular joint, temporomandibular joint disorders, structured data entry, dentistry, data model, text-to-speech system, Research Diagnostic Criteria for TMD

\section{Introduction}

In all fields of medicine the amount of different types of information is increasing. It is highly necessary to have complex information about patient's health status (history, investigation and therapy data). It is also important that this information is always available and stored in a comprehensive form. All these requirements can be fulfilled only by modern computer systems especially by the Electronic Health Record (EHR). Therefore the need of adopting a suitable EHR system (EHR-S) is gradually increasing. However, various studies reveal conflicting data regarding time effectiveness on workflow due to computerization. One of the major issues for physicians is to evaluate potential impact of EHR-S on workflow [1]. Another major issue for the purpose of establishing EHR-S is business-oriented health data distribution in different systems, which should be integrated to focus on individuals [2]. Generally, EHR-Ss should support continuous, efficient and highquality integrated healthcare by using structured data entries [3], [4], interoperability [5] and standards [6]. At the beginning of the development of different EHR-Ss the International standard ISO/DTR 20514:2005 was published and defined the EHR as a repository of information regarding the health of a subject of care in a computer process able form, stored and transmitted securely, and accessible by multiple authorized users. The same process of the computerization has been started also in the dentistry. It was performed by various systems. They offered many graphical approaches, but none of them is supported by an automatic speech recognition system. Voice commands usage has been experimented since 1990's [7] as a convenient replacement of a computer control. The necessity of using a human voice to control a computer or another device arises in typical hands-busy environments such as surgery or dentistry [8].

Another challenge is the real complexity of the system. This leads to the creation of the supplementary EHR-S, which is concerned with temporomandibular joint issues and consequently covers the whole orthognathic system.

TMD is an umbrella title for symptoms as temporomandibular dysfunction, craniomandibular disorder and mandibular dysfunction. TMD includes a variety of conditions associated with pain and dysfunction of the temporomandibular joint and masticatory muscles [9]. An estimated $20 \%$ of the population is affected, with $10 \%$ to $20 \%$ of those seeking treatment [10], [11]. 
The main symptoms are pain in the masticatory muscles, limitations or deviation of the mouth opening and TMJ sounds. The etiology of the TMD is multifactorial, therefore detail and complex information is for the therapy indication very important. The diagnosis of TMD is commonly based on the presenting sings and symptoms. For the classification among clinicians and researchers The Research Diagnostic Criteria for the Temporomandibular Disorders (RDC/TMD) are used. According to the mentioned facts, our main goal is to develop an EHR focused on the TMD, which will lead to the decision support system in the therapy of TMD.

\section{Methods \\ 2.1 MUDR EHR, Dental Knowledge database and MUDRLite}

The EuroMISE Centre has a long tradition in research and development of EHR-Ss, which started in the year 2000. The research resulted in a pilot EHR application called MUDR (Multimedia Distributed EHR). The main requirement for the system was the storage of structured data combined with free text with the possibility of dynamic extension and modification of the set of collected attributes without any change of the database structure. The MUDR EHR was based on 3-tier architecture, consisting of a database layer, application layer and user interface. The set of definitions of collected attributes and relations between them is stored in a directed graph structure called "knowledge base".

The modelling tools were developed as a part of MUDR EHR and were employed in this project to create a Dental Knowledge Base. This knowledge base was created in cooperation between IT specialists and dentists in years 2004 and 2005 . It comprises of approximately 1000 concepts that cover all possible situations that we can find in the patient's dental status and basic facts about the patient as well.

Since the MUDR EHR was designed for large scale enterprises, which was in some cases a limiting factor, another EHR-S was developed at the EuroMISE Centre in 2005. This EHR-S was related to MUDR EHR, hence named MUDRLite [12] and was developed with the emphasis on the small practice needs. The MUDRLite architecture is based on two tiers. The first one is a relational database (e.g. MS SQL server) and the second one is a MUDRLite User Interface layer (MUDRLite UI). The database schema corresponds to the particular needs of the target domain and therefore varies significantly in different environments. The core of MUDRLite MUDRLite Interpreter is able to handle various database schemas. This feature often simplifies the way of importing old data stored in other databases or files. The visual aspects as well as the behaviour of the MUDRLite Ul are completely described by an XML configuration file. The end-user can see a set of forms with various controls placed on them by appropriate XML elements.

MUDRLite operates as a command interpreter; it processes the instructions encoded in the so called MLL (MUDRLite Language) [13] based on XML and manipulates the database layer as well as the visual aspects and behaviour of the MUDRLite user interface. The pilot application was prepared for dentistry [8] and as a next step for the TMD, which will be mentioned below.

\subsection{DentCross component, ASR}

EHR systems MUDR and MUDRLite were usable for the general clinicians. In the field of dentistry it is common to have information presented in the graphical form. For this reason and for better user acceptance an advanced component representing the dental cross was developed, which is clearly organized in the graphical form. The DentCross component is implemented as a standalone library DentCross.dll, completely developed for the .NET Framework platform. This user-defined component is inserted by the custom element of the MLL (named "custom") with the following mandatory attributes: "dll" specifying the name of the assembly where the component is implemented and "class" specifying the name of the main class of the included component.

The user interface for collection of the TMD data is a user form defined completely in MLL. It is integrated in the same application as the DentCross is, thus creating a comprehensive EHR in dentistry [14].

Real interactivity was achieved by adoption of the system for the automatic speech recognition and the text to speech module. The automatic speech recognition (ASR) engine is described in more detail in [15] and [16].

\subsection{RDC/TMD}

In the specialized part of the EHR aimed on the TMD we have used basic schema for the anamnesis. For the TMD classification we have used parts of RCD/TMD, which applies the dual-axis system to diagnose and classify patients with TMD [17]. The first axis is divided into 3 groups of commonly occurring TMDs:

1. muscle disorders, including myofascilal pain,

2. disk displacement with or without reduction,

3. arthralgia, arthritis and arthrosis.

The second axis in this nomenclature is using the 31-items questionnaire, used to evaluate behavioural, psychological and psychosocial factors. We have modified these investigated factors and we have created our own questionnaire for evaluation of the quality of life.

\section{Results}

A knowledge modelling tool from the MUDR EHR application suite was used to create a specialized TMD Knowledge Base. This knowledge base contains more than 80 concepts (Fig. 1) and extends the former Dental Knowledge Base.

In order to create MUDRLite forms, concepts from the TMD Knowledge Base had to be transformed in a relational database model (Fig. 2). The user interface and application for TMD was encoded into MLL in MUDRLite EHR, thus creating user forms interconnected with a database layer.

The basic part of our interactive EHR in dentistry is composed of the DentCross component with the ASR system, which is built on concepts originating from the Dental Knowledge Base and uses the MUDRLite EHR as a hosting system. The specialized TMD EHR contains concepts defined in the TMD Knowledge Base. 


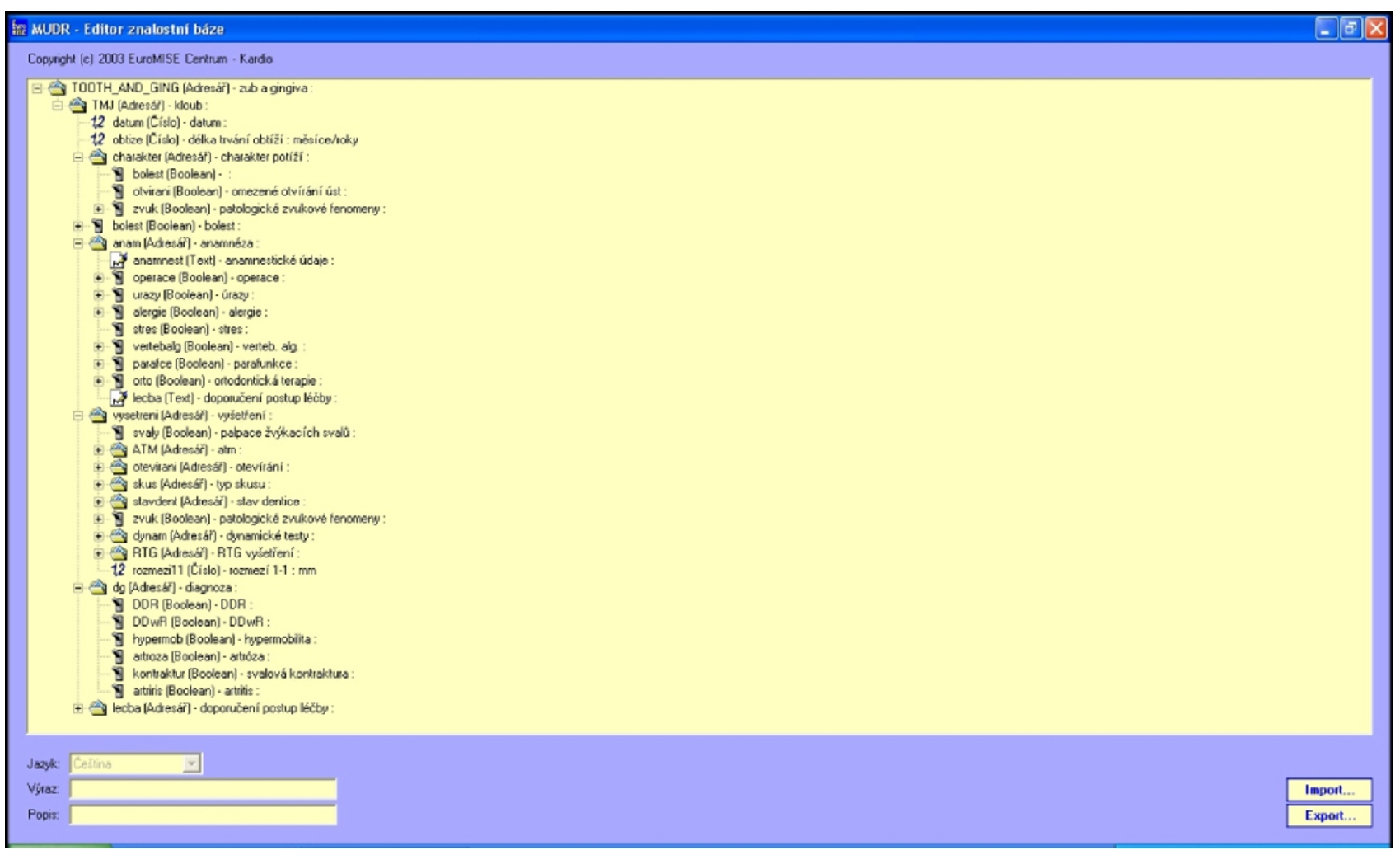

Fig. 1. TMD knowledge base.

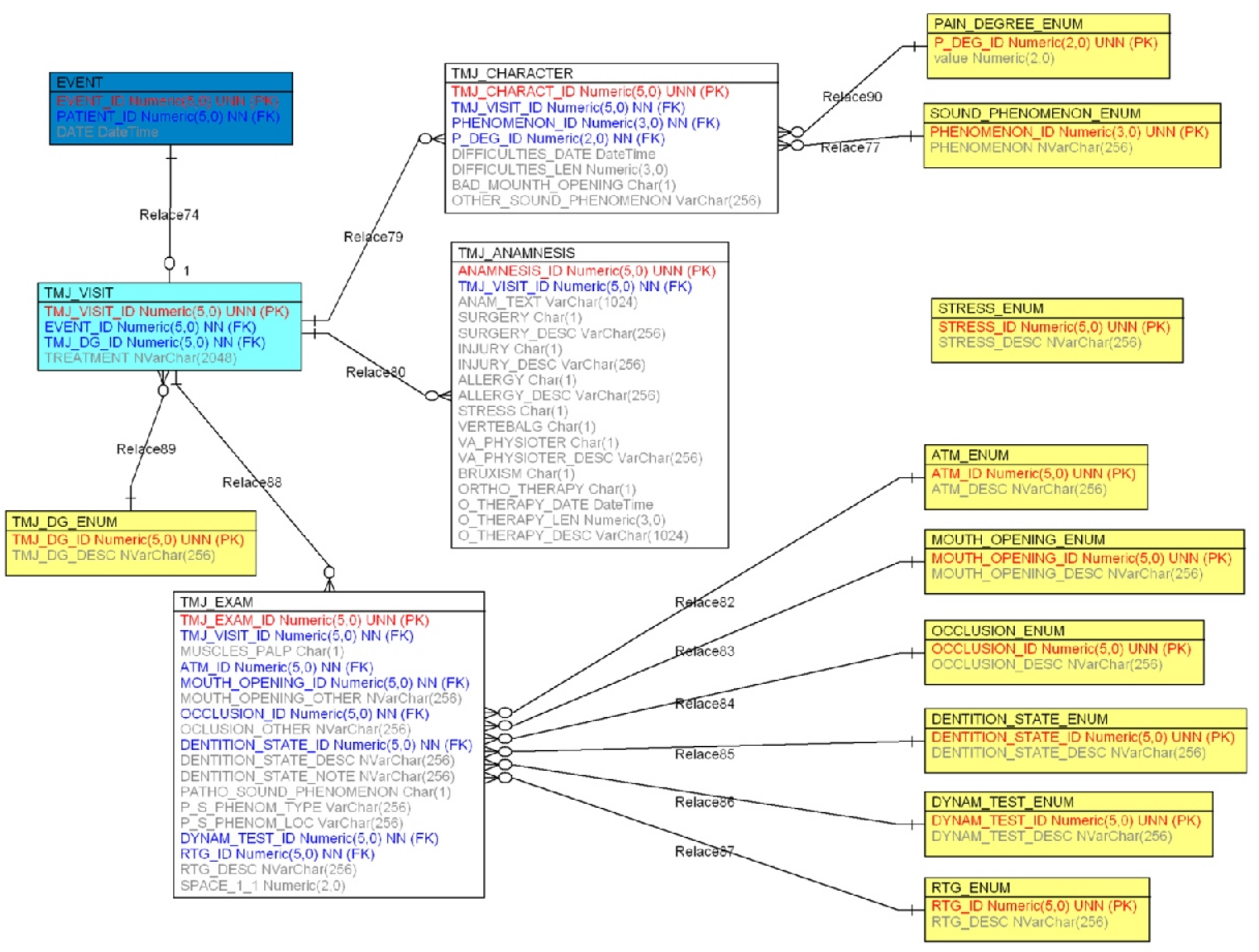

Fig. 2. Data model for TMD EHR and its tree-structure. 
It is initiated from the main window of the Integrated Dental-TMJ EHR (DentCross Component, ASR, TTS and TMD part) and has its own user interface.

This application consists of 3 main parts (Fig. 3). In the first part we can find basic patient facts (name, id number, date of birth etc.), which helps in orientation. The second part is the list of events. They are sorted by the dates and by opening these dates we can reach and open records connected with one particular patient visit. The third part consists of the subjective patient problems, anamnestic data, results of investigation, $\mathrm{dg}$ statement and therapy recommendations. This part is the most important and represents all data about the particular patient.

It is very important to know, what circumstances lead a patient to visit the specialist and what patient's personal feelings are. The clinician can recognize many important facts from the patient description. We can record a type and character of difficulties, their duration and closer description. In the anamnestic section we can record basic personal anamnestic data and different conditions important to the TMD (parafunctions, stress, orthodontic treatment).
Probably the most important is the section with investigation modalities. Investigation is in the TMD classified in aspection (deformities, status of dentition, mouth opening (Fig. 4) etc.), palpation (masticatory muscles, TMJ) and auscultation (TMJ sounds). The last part in the TMD investigation playing the crucial role is X-ray imagining (ortopantomographic image, computer tomography (Fig. 5)) and magnetic resonance. Subscription and presence of these images can be also recorded. In the diagnosis classification we have used above mentioned RDC/TMD. We can record also therapy recommendation, which can be changed at the end of each visit. This application serves as a complex information source for the TMD treatment and is a part of the whole system for the support in decision in the therapy of TMD, which leads in the final treatment plan:

1. implementation to the interactive DentCross component - dental examination and anamnestic data,

2. training of opening of the mouth in the axis, without deviation,

3. stabilization splint,

4. computer-aided face bow analysis (Fig. 6),

5. prosthetic treatment, 6.finalization of the treatment and soft splint,

7.recall and treatment evaluation by the patient.

\section{Discussion}

The presented DentCross component especially with the specialized part for the TMD and ASR system seems to be highly suitable for the field of dentistry. Its graphical form offers a good overview during patient's investigation and treatment. Voice commands and the system for ASR enable control of the data recording without other person's assistance. Another advantage is the possibility of data recording without a traditional control by a mouse and keyboard. This is very important especially in the dentistry and medicine in general. A hand with the glove is in the permanent contact with the potentially infectious material. The DentCross component is used in the dental office in the Department of paediatric stomatology, Faculty hospital Motol and was used in the Department of biological analysis Institute of Criminalistics in Prague for dead corpses' identification [8].

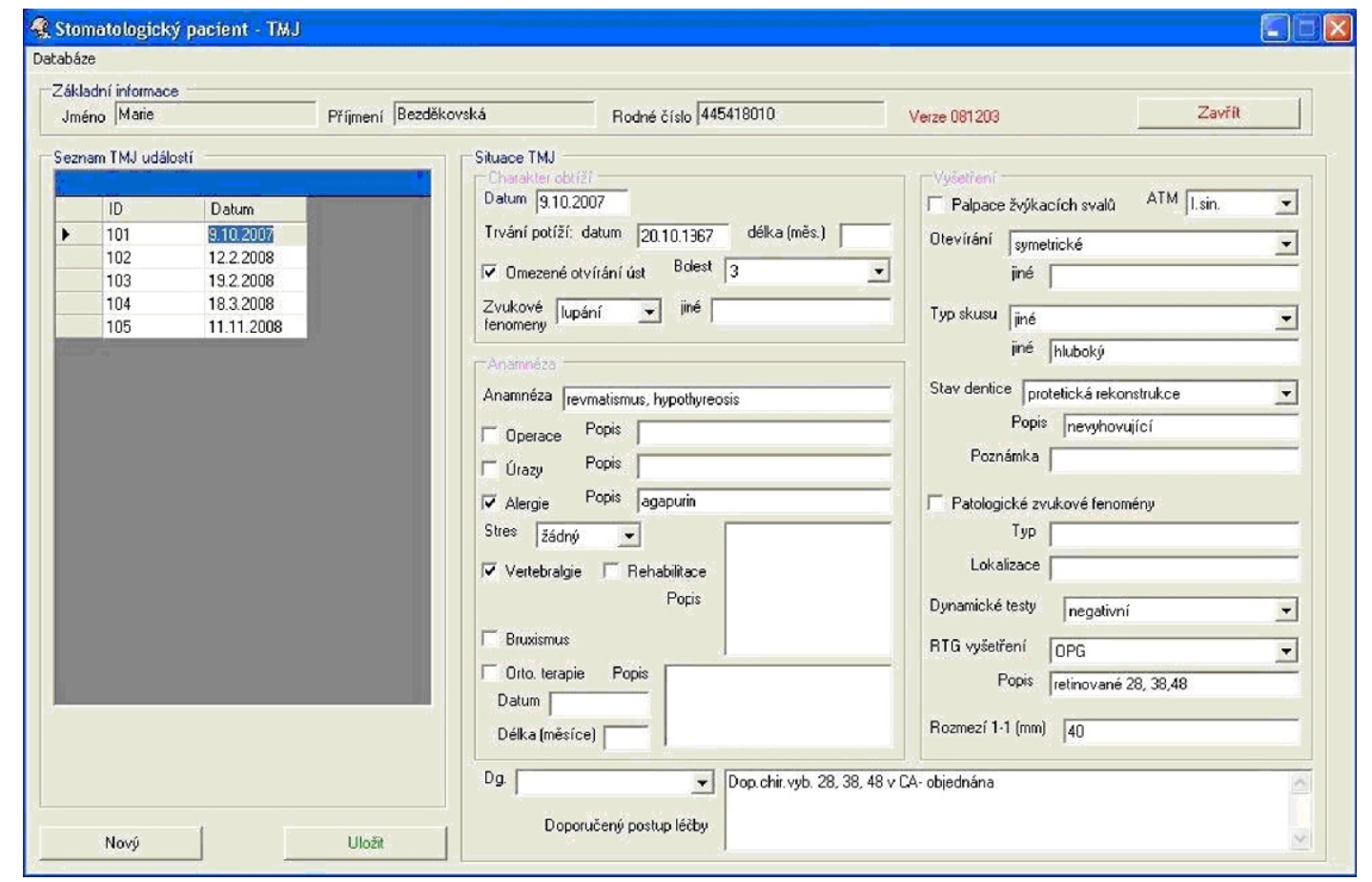

Fig. 3. TMD EHR application. 
The specialized TMD part of the Integrated Dental - TMJ EHR extends the contemporary EHR system in the dentistry. It consists of basic anamnestic data, investigation data, diagnose statement and therapy recommendation. This application was used in the special office at the Faculty hospital Motol for the therapy of the TMD and was a part of the system for the decision support in the therapy of TMD. It allows a complex overview during investigation, treatment planning and it also saves some time.

The future work is to improve the Integrated Dental-TMJ EHR and create an automatic system built on the anamnestic data signs and symptoms to make a diagnosis and prepare a therapeutic plan. This would facilitate the whole therapy process of the multifactorial conditioned TMD.

\section{Acknowledgments}

This work was supported by the Grant 9991-3 IGA Ministry of Health CR, project FI-IM5/142, 1 M06014 Ministry of Education, Youth and Sports and SVV2010-265513.

\section{References}

[1] Shabbir S.A., Ahmed L.A., Sudhir R.R. Scholl J., Li Y.C., Liou D.M.: Comparison of documentation time between an electronic and a paper-based record system by optometrist at an eye hospital in south India: A time.motion study. Comput Methods Programs Biomed, 2010 May 10.

[2] Liu D., Wang X., Pan F., Yang P., Xu Y., Tang X., Hu J., Rao K.: Harmonization of health data at national level: a pilot study in China. Int J Med Inform, 2010 Jun; 79(6): 450-8.

[3] Los R.K., van Ginneken A.M., van der Lei J.: OpenSDE: a strategy for expressive and flexible structured data entry. Int J Med Inform 2005; 74:481-490.

[4] van Ginneken A.M.: The computerized patient record: balancing effort and benefit. Int J Med Inf 2002; 65:97-119.

[5] Bakker A.: Access to EHR and access control at a moment in the past: $A$ discussion of the need and an exploration of the consequences. Int J Med Inform 2004; 73:267-270.

[6] Blobel B.: Advanced EHR Architecture Promises or Reality. Methods Inf Med 2006; 1: 95-101.

[7] Grasso M.A.: Automated speech recognition in medical applications. MD Computing 1995; 12: 16-23.

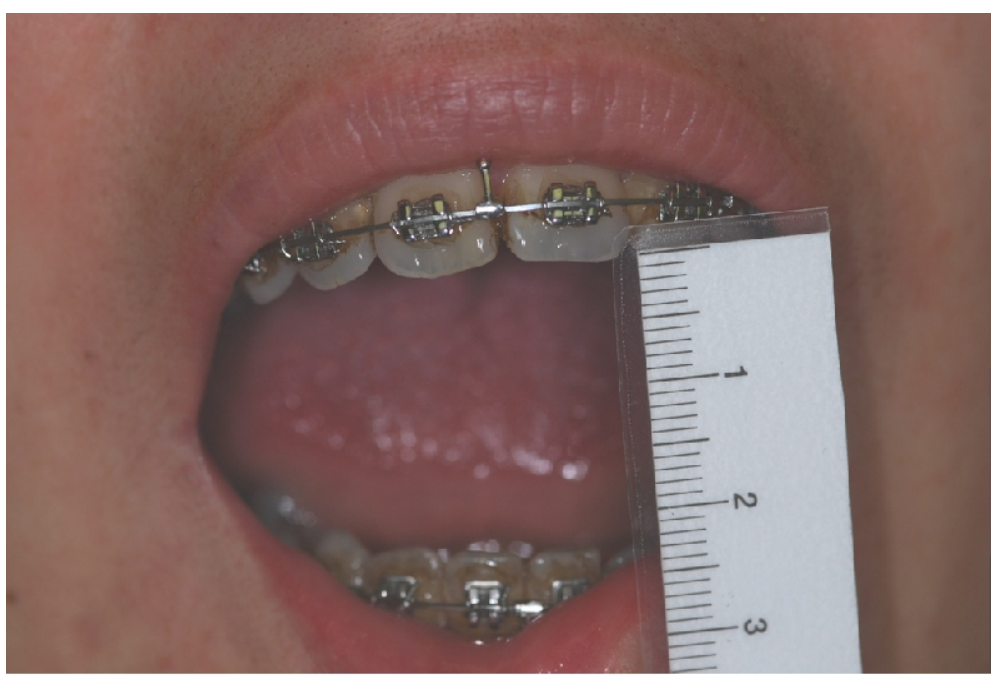

Fig. 4. TMJ - data collection. Measurement of the mouth opening.

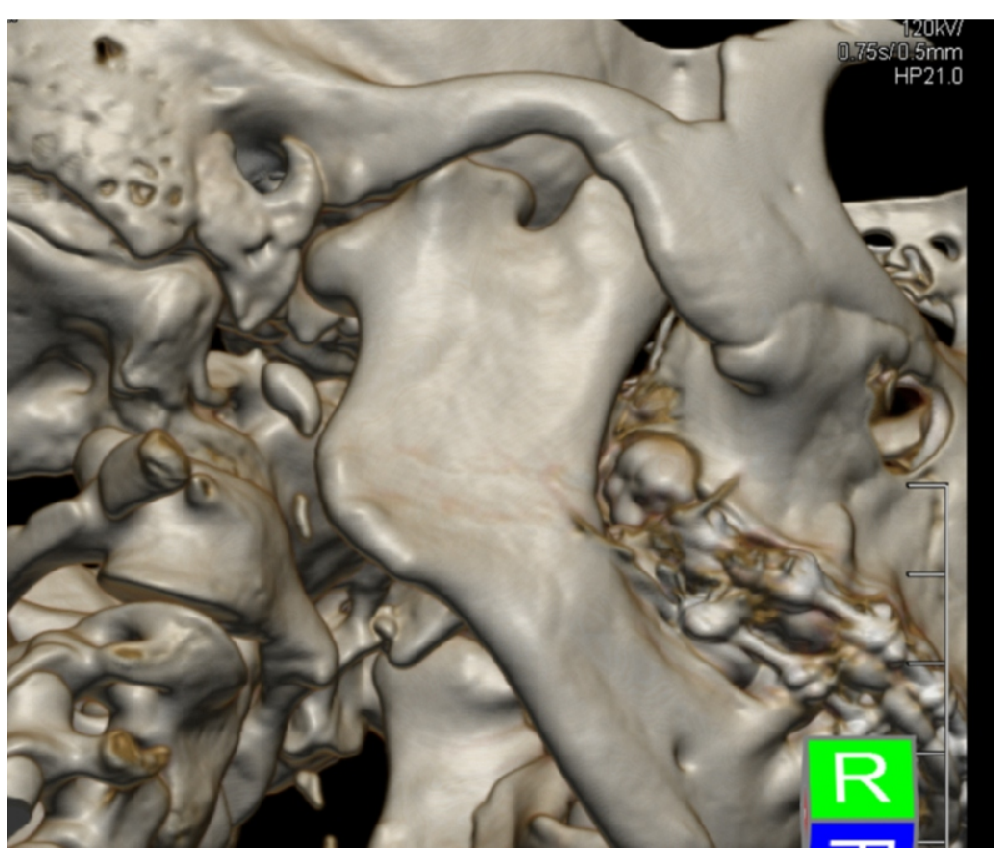

Fig. 5. TMJ - 3D-CT computer image analysis. In this picture is presented TMJ ankylosis.

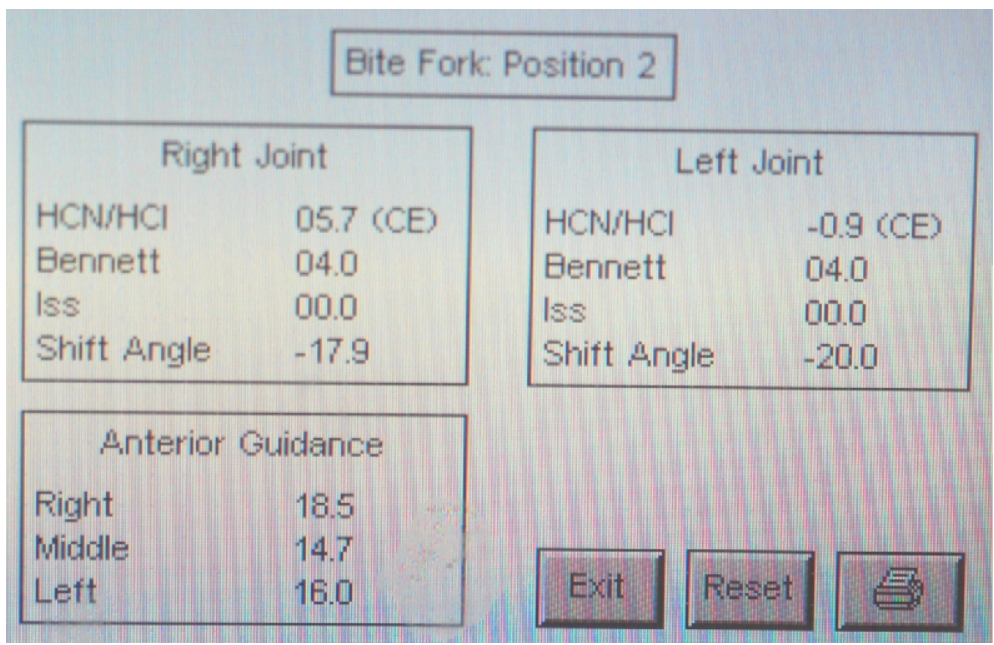

Fig. 6. TMJ - computer face bow analysis. 
[8] Zvarova J., Dostalova T., Hanzlicek P., Teuberova Z., Nagy M., Pies M., Seydlova M., Eliasova H., Simkova H.: Electronic health record for forensic dentistry. Methods Inf Med 2008; 47: 8-13.

[9] Di Fabio R.P.: Physical therapy for patient with TMD: a descriptive study of treatment, disability and health status. J Orofac Pain. 1998;12:124-135.

[10] Pedroni C.R., De Oliveira A.S., Guaratini M.I.: Prevalence study of signs and symptoms of temopomandibular disorders in university students. J Oral Rehabil. 2003; 30: 944-950.

[11] Taylor M., Suvinen T., Reade P.: Teh effect of Grade IV distraction mobilization on patients with temporomandibular paindysfunction disorder. Physitherapy Theory and Practice. 1994;10:129-136.

[12] Hippmann R., Dostalova T., Zvarova J., Nagy M., Seydlova M., Hanzlicek P., Kriz P., Smidl L., Trmal.: Voice supported electronic health record for temporomandibular joint disorders. Methods of information in medicine, 2010; 49:168-172

[13] Spidlen J., Hanzlicek P., Zvarova J.: MUDRLite - health record tailored to your particular needs. Studies in health technology and informatics 2004; 105: 2029.

[14] Hippmann R., Dostalova T., Zvarova J., Nagy M., Seydlova M., Hanzlicek P., Kriz P., Smidl L., Trmal.: Voice supported electronic health record for temporomandibular joint disorders. Methods of information in medicine, 2010; 49:168-172.

[15] Nagy M., Hanzlicek P., Zvarova J., Dostalova T., Seydlova M., Hippmann R., Smidl L., Trmal J., Psutka J.: Voicecontrolled Data Entry in Dental Electronic Health Record. Studies in Health Technology and Informatics 2008; 136: 529534.
[16] Psutka J., Muller L., Matousek J., Radova V.: We speak Czech with a computer Praha, Academia, 2006.

[17] Ali H.N.: Diagnostic criteria for temporomandibular joint disorders: a physiotherapist's perspective. Physiotherapy. 2002; 88:421-426.

\section{Contact \\ MUDr. Radek Hippmann}

Department of Paediatric Stomatology, Second Faculty of Medicine of Charles University

Vúvalu 84

15006 Prague 5

Czech Republic

e-mail:r.hip@seznam.cz 